\title{
El cliente en psicoterapia: contribución al resultado terapéutico
}

\author{
Alberto Rodríguez-Morejón \\ Facultad de Psicología. Universidad de Málaga (España).
}

\begin{abstract}
Resumen: En este trabajo se revisan las evidencias empíricas que apuntan a que los clientes son los principales responsables de los resultados de los tratamientos. Para ello se presentan dos tipos de datos: a) los que demuestran que las personas se enfrentan exitosamente a los problemas sin ayuda: estudios sobre la resiliencia, la recuperación espontánea, la auto-ayuda o el efecto placebo; y b) los que avalan que los usuarios de una psicoterapia son agentes activos durante todo el proceso terapéutico. Finalmente se hacen sugerencias sobre cómo adaptar las prácticas terapéuticas a esta nueva visión de los clientes.

Palabras clave: Investigación en psicoterapia; resultados terapéuticos; clientes en psicoterapia; relaciones de colaboración.
\end{abstract}

\section{Introducción}

¿Por qué funciona la psicoterapia? Una respuesta que muchos profesionales aceptarían podría ser la siguiente: un terapeuta bien entrenado, hace una buena evaluación de un usuario con problemas, la evaluación conducirá a un diagnóstico, y éste ayudará a diseñar un tratamiento concreto. Si la terapia se lleva a cabo adecuadamente las probabilidades de éxito aumentarán.

En esta descripción clásica del proceso de evaluación y tratamiento, el terapeuta es claramente el protagonista del trabajo. De modo que cabría pensar que si todos los pasos anteriores se hacen adecuadamente, el resultado de la intervención está casi garantizado. Pero, ¿cuál es la contribución de los clientes?, ¿son los invitados de piedra en este proceso?, ¿son realmente los terapeutas y sus técnicas los protagonistas absolutos de esta historia? Todas esas preguntas tienen muchas respuestas posibles, pero la tesis que se defiende en este artículo es clara: la psicoterapia es mucho más que un terapeuta y sus técnicas, la contribución de los clientes es esencial. El objetivo de este trabajo es demostrar que ésta afirmación está además sostenida por evidencias empíricas.

\section{Las variables del proceso terapéutico}

¿Qué variables hay que tener en cuenta para explicar el éxito o fracaso de una terapia? Por un lado está la contribución de las personas: clientes (con sus características diferenciales) y terapeutas (con su experiencia y habilidades comunicativas). A ellas hay que añadirles el efecto debido al enfoque terapéutico que usa el clínico (con sus presupuestos teóricos, técnicas y criterios para tomar decisiones de proceso terapéutico). Todas estas variables hay que entenderlas siempre en interacción: cliente y terapeuta forman un equipo, el pri-

* Dirección para correspondencia [Correspondence address]: Alberto Rodríguez Morejón. Universidad de Málaga. Facultad de Psicología. Campus de Teatinos S/N. CP 29071. Málaga (España). Email: aromore@uma.es
Title: Clients in psychotherapy: contribution to therapeutic outcome. Abstract: In this paper the empirical evidence pointing that customers are primarily responsible for the results is reviewed. Two types of data are presented: (a) those which demonstrate that people successfully face problems without help: studies on resilience, spontaneous recovery, self-help or placebo effect; and (b) those which show that the users of a psychotherapy are active agents throughout the therapeutic process. Finally, suggestions are made on how to adapt the therapeutic practices to this new vision.

Key words: Psychotherapy research; psychotherapeutic outcome; client agency; collaborative relationship; client factors.

mero es especialista en su problema y el segundo en resolver problemas; el trabajo del terapeuta es adaptar los tratamientos a las necesidades y características de los usuarios, así como crear una buena relación terapéutica sobre la que poner en marcha las técnicas de cambio.

Esta visión de la terapia como un trabajo colaborativo encaja mucho mejor con los datos de investigación que la visión del "terapeuta omnipotente" con la que se iniciaba este apartado.

\section{La contribución de cada variable}

Descritas las variables, se especificará la contribución de cada una de ellas a la varianza de resultados. El primer intento de crear un modelo explicativo se lo debemos a Lambert, Shapiro y Bergin (1986). Desde su enfoque de factores comunes, la varianza del éxito en psicoterapia se explicaría por cuatro variables: factores extra-terapéuticos del cliente (40\%); relación terapéutica (30\%); placebo, expectativas y esperanzas de cambio (15\%); y modelo/técnicas (15\%).

En la misma línea Wampold (2001), usando una metodología meta-analítica, concluye que el $87 \%$ de la varianza de cambio corresponde a factores relacionados con el cliente y tan sólo el 13\% se relacionaría con la terapia, o mejor dicho, con la contribución directa de la técnica o el terapeuta. Un modelo más reciente reparte el $13 \%$ atribuido a los tratamientos de la siguiente manera: el terapeuta sería el responsable de entre el 6 y el 9\%, la alianza terapéutica de entre el 5 y el 7\% y el modelo/técnica del 1\% (Duncan, 2010).

La cuestión es que a partir de estos datos difícilmente se puede mantener la idea de que los terapeutas y sus técnicas son los protagonistas del proceso. Parece claro que la contribución de los clientes al resultado final de la psicoterapia es, al menos cuantitativamente, más decisiva (Duncan y Miller, 2000). En los siguientes apartados se reflexionará sobre esta idea: se presentarán evidencias empíricas para demostrar que las personas son solucionadores de problemas activos y eficaces en su vida cotidiana, y que esta actitud se mantiene cuando son usuarios de una psicoterapia. 


\section{Los clientes: héroes o villanos}

Si los datos de los meta-análisis que se han presentado apuntan a la importancia de la contribución de los usuarios, ¿por qué hasta ahora se ha mantenido esa visión tan negativa del ser humano que acude a consulta? Tal vez se deba a una excesiva generalización a partir del tipo de casuística que constituía el objeto de trabajo de los primeros especialistas en lo mental. Es cierto que las personas aquejadas por trastornos mentales tan graves como las psicosis pueden encajar más en ese papel de enfermos desvalidos que un especialista debe curar. Sin embargo, la mayoría de las personas que reciben psicoterapia asumen papeles muy diferentes en sus tratamientos. Son individuos que, por diferentes razones, no consiguen enfrentarse a las dificultades que les imponen sus vidas, o son incapaces de controlar algunos aspectos de ellos mismos que no les gustan o no funcionan, y buscan ayuda para salir adelante. $\mathrm{Y}$ eso es lo que como especie llevamos haciendo decenas de miles de años: resolver problemas cambiando el entorno que nos rodea o cambiándonos a nosotros mismos para adaptarnos al contexto cuando no podemos modificarlo.

El entender que las personas son héroes luchando en un contexto difícil o villanos atrapados en las limitaciones de su historia y sus déficits de personalidad o biológicos, ha sido hasta ahora una cuestión de decisión personal. Elección, por lo demás, muy asociada a la orientación terapéutica que como profesional cada uno decide asumir. Hay enfoques de psicoterapia que hacen descripciones muy negativas de las personas y sus familias, entendiendo los problemas en términos de patología familiar y personal. Otros modelos en cambio tienen una visión más positiva del ser humano, buscan sus lados fuertes y construir cambios desde sus recursos. Es muy probable que tanto las visiones más negativas como las más positivas puedan sustentarse en evidencias empíricas. El objetivo del análisis que se emprende en este escrito es demostrar que entender a los clientes como competentes y activos, siendo una elección, es sin embargo una práctica basada en la evidencia (Bohart y Greaves, 2013; Bohart y Tallman, 1999; Duncan y Miller, 2000).

Dos tipos de investigaciones soportan la premisa de que las personas tiene recursos y son expertos en resolver problemas (Bohart y Tallman, 2010): a) las que demuestran que las personas son capaces de enfrentarse a los problemas psicológicos sin ayuda de especialistas y b) las que apuntan a que los usuarios de una psicoterapia son agentes activos y altamente implicados en el proceso de cambio.

\section{Los seres humanos se enfrentan eficazmente a sus} problemas

Nuestra especie no seguiría sobre el planeta si no fuéramos capaces de eso. Los humanos somos expertos en resolver problemas y lo demostramos cuando las cosas son muy complicadas y tenemos que sobrevivir en ambientes muy difíciles o cuando sufrimos catástrofes inesperadas y debemos luchar al límite para sobreponernos o cuando padecemos enfermedades físicas o mentales a las que tenemos que hacer frente. Para repasar las evidencias a favor de esa tesis se hará referencia a cuatro fuentes: los estudios sobre la resiliencia, la recuperación espontánea, el impacto de la auto-ayuda o la respuesta placebo.

\section{La resiliencia}

La resiliencia es uno de los temas de moda en este momento de auge de la psicología positiva. La capacidad de algunos niños para resistir y sobreponerse a ambientes desfavorecidos (Zolkoski y Bullock, 2012) o incluso a tremendos desastres (Masten y Narayan, 2012) está bien establecida. Un trabajo paradigmático en esa línea es el de Werner y Smith (1989). Se trata de un estudio longitudinal en el que hacen un seguimiento a todos los niños nacidos en una isla hawaiana en el año 1955. Las autoras encuentran que el 66\% de los que crecen en los ambientes más desfavorecidos tienen problemas de conducta y escolares a los 10 años, y legales o de salud mental a los 18. Sin embargo, a los 40 años casi todos se han convertido en adultos perfectamente integrados.

La literatura está llena de evidencias parecidas. George Vaillant (1993) en su obra The Wisdom of the Ego, narra un seguimiento de 35 años de un grupo de hombres. El 64\% de los que a los 24 años eran considerados como "casos sin solución" por diversas causas, a los 60 estaban en el cuartil más alto de indicadores de salud mental.

En definitiva, los estudios sobre resiliencia demuestran que muchas personas salen adelante en contextos difíciles e incluso superando situaciones catastróficas, lo que parece una evidencia clara a favor de la tesis de este trabajo: las personas tienen recursos para enfrentarse a los problemas.

\section{La recuperación espontánea}

Casi todos los seres humanos son capaces de resolver problemas psicológicos sin ayuda de especialistas. El 90\% de las personas afirman que han tenido que superar problemas importantes de salud o emocionales a lo largo de su vida, y la mayoría lo hacen sin la ayuda de un profesional (Bohart y Tallman, 2010). ¿Pero y qué pasa cuando los problemas no son los habituales de la vida cotidiana y las personas se enfrentan a lo que se puede considerar trastornos psicológicos graves? Pues que contamos con pruebas de que las personas también superan con dignidad trastornos tan relevantes como la conducta antisocial (Tedeschi y Felson, 1994), las adicciones (Miller y Carroll, 2006) o los trastornos de la personalidad (Skodol et al., 2007).

La investigación sobre estrés postraumático aporta datos relevantes que apoyan la fortaleza con la que somos capaces de enfrentarnos a las adversidades. Casi la mitad de las personas sufren en algún momento de su vida un acontecimiento altamente traumático y de ellas sólo entre el 5 y el $10 \%$ acaban sufriendo algún tipo de estrés postraumático (Ozer, 
Best, Lipsey y Weiss, 2003). Además, entre el 40 y el 60\% de las personas que llegan a padecer este tipo de trastornos se recuperan por sí solas e informan que la experiencia les ha servido como crecimiento personal y para hacerse más fuertes (Calhoun y Tedeschi, 2006).

Uno de los últimos meta-análisis sobre recuperación espontánea en depresión ofrece datos reveladores a este respecto. Whiteford et al. (2013) revisan los 21 estudios que analizan la evolución de listas de espera de personas diagnosticadas de depresión mayor para establecer el índice de recuperación espontánea en este trastorno. Encuentran que el $23 \%$ de las personas se recuperan sin ayuda en tres meses, el $32 \%$ en seis, y la cifra a un año de seguimiento llega hasta el $53 \%$. Esto es, más de la mitad de las personas superan una depresión mayor por sí solas en el plazo de un año, lo cual parece una prueba bastante sólida de la capacidad humana para recuperarse de problemas psicológicos graves.

\section{El impacto de la autoayuda}

Otra evidencia de las capacidades auto-curativas de las personas es el impacto que tienen los libros y programas de auto-ayuda. Las librerías están llenas de libros que ofrecen diferentes alternativas de auto-sanación para los problemas psicológicos. Por ejemplo, algunos estudios encuentran que la eficacia de la biblioterapia se aproxima a la de la psicoterapia (Norcroos, 2006). La auto-ayuda funciona, pero ¿cómo de eficaz es cuando se la compara con la psicoterapia? En principio cuando se comparan ambos tipos de intervenciones se encuentran diferencias significativas a favor de la psicoterapia (Menchola, Arkowitz y Burke, 2007), concluyéndose que los tratamientos con terapeuta se hacen necesarios sobre todo cuando los problemas son más graves.

En cambio, el último gran meta-análisis de Cuijpers, Donker, van Straten, Li y Andersson (2010) encuentra justo lo contrario. Estos autores revisan 21 estudios en los que se comparan tratamientos de auto-ayuda con psicoterapia "cara a cara". En todos ellos se asigna al azar a personas con ansiedad o depresión a un tratamiento de psicoterapia tradicional o a un tratamiento de auto-ayuda guiada diseñado por un profesional. Sus análisis muestran ligeras diferencias a favor de los tratamientos auto-aplicados a terminación y ninguna diferencia entre las dos modalidades en seguimiento.

En definitiva que, a pesar de que los datos no son concluyentes, sí parece claro que los tratamientos auto-aplicados tienen resultados aceptables, y no muy diferentes a los de un tratamiento "cara a cara" con un terapeuta. Una evidencia más del potencial de cambio de las personas.

\section{La respuesta placebo}

En la misma línea de razonamiento, hay que revisar los sorprendentes efectos que el placebo tiene sobre la salud de las personas. Un meta-análisis de Grissom (1996) demuestra que los tratamientos placebo (sin supuestos ingredientes terapéuticos) consiguen variaciones significativas en los pro- blemas psicológicos en comparación con el no tratamiento. El revolucionario trabajo de Kirsch demuestra algo parecido incluso para los tratamientos biológicos. Sus datos mostraron que los antidepresivos no tienen más poder terapéutico que un placebo en depresiones leves y moderadas (Kirsch et al., 2008), lo que llevó a afirmar que el 75\% del efecto de algunos antidepresivos es puro placebo (Kirsch, 2010). El mismo autor defiende que el componente de placebo que tienen las psicoterapias es indudable (Kirsch, 2005).

Un ejemplo potente de lo que consigue el placebo lo encontramos en el estudio de Kaptchuk et al. (2008). Ellos demuestran que las palabras que acompañan a la prescripción de un medicamento influyen significativamente en los efectos de este. A un conjunto de pacientes con Síndrome de intestino irritable se les asigna al azar a tres grupos: a) lista de espera, b) un tratamiento placebo, o c) placebo con manejo clínico. En este último caso, una profesional dedica un tiempo a hablar con el paciente de sus síntomas, a interesarse por su sufrimiento y crear expectativas de éxito. El tercer grupo es el que experimenta un mayor alivio de los síntomas. Además resulta que su mejoría es equivalente a la que producen las dos medicaciones más utilizadas para el tratamiento del síndrome, y que los cambios se mantienen en el tiempo.

El que las palabras pronunciadas por los terapeutas tengan efectos de cambio tan potentes en las personas parece una cuestión de mucho interés para la psicoterapia. Con el valor añadido de que conocemos cada vez más sobre cómo funcionan los placebos. Desde un punto de vista cognitivo la respuesta placebo parece estar mediada por la creación de expectativas, los cambios se producen sólo cuando las personas son capaces de imaginarse un futuro sin el problema (Benedetti et al., 2006). Los estudios han logrado, además, clarificar que la respuesta fisiológica asociada al placebo consiste en un aumento de la producción de dopamina que bloquea las hormonas del estrés, relaja y permite un mejor rendimiento cognitivo (Benedetti, 2013)

Resumiendo. Tenemos que algunas personas son capaces de sobreponerse y resistir a los ambientes más difíciles, que pueden recuperarse de trastornos tan graves como la depresión o el estrés postraumático, que son capaces de beneficiarse de programas de auto-ayuda con muy poco apoyo de un terapeuta y que tienen potentes respuestas positivas ante estímulos que les ayudan a creer en que los cambios son posibles. Esas personas, como se expone en el siguiente apartado, cuando participan en un tratamiento psicológico, son también activas y colaboradoras.

\section{Los usuarios participan activamente en psico- terapia}

Las personas suelen tener sus propias ideas sobre a qué se deben sus dificultades y sobre qué tiene que pasar para que se resuelvan. También los modelos de psicoterapia ofrecen explicaciones para entender los síntomas como paso previo 
a proponer tratamientos para hacerlos desaparecer (Frank, 1973). ¿Cómo conjugar las explicaciones de unos y de otros? Ahí radica precisamente la clave del ajuste en psicoterapia, en conseguir un compromiso entre las ideas que el cliente trae y las nuevas visiones que el terapeuta tratará de generar. En este apartado se exponen algunas evidencias que hacen pensar que, en este proceso, el usuario es un agente intencional y activo. Para revisar las investigaciones se hará referencia a tres momentos del tratamiento: el inicio, las sesiones y los periodos entre sesiones.

\section{Preferencias, cambios y expectativas}

La mejor demostración de que los usuarios no son receptores pasivos de la psicoterapia es que en un 60\% de los casos traen cambios pre-tratamiento (Lawson, 1994); esto quiere decir que han empezado a hacer algo diferente respecto al problema que ayuda a que vengan con algún tipo de mejoría a la primera sesión. En esta misma línea parece sorprendente que en torno a un $30 \%$ de las personas informen sobre mejorías en la segunda sesión, cuando se supone que todavía los tratamientos no han tenido tiempo de hacer mucho efecto (Howard, Kopta, Krause y Orlinsky, 1986).

Los usuarios tienen preferencias sobre qué condiciones del encuentro terapéutico se acomodan más a sus intereses. Ideas sobre el terapeuta que necesitan (sexo, edad, cultura), sobre en qué debe consistir el tratamiento (formato, enfoque) o el tipo de actividades que convendría realizar. Un meta-análisis en el que se repasan los estudios que comparan asignación forzosa a un terapeuta o asignación en función de las preferencias, demuestra que cuando las personas eligen su terapeuta hay entre un 35 y un 50\% menos de abandonos y mejores resultados finales (Swift, Callahan y Vollmer, 2011).

Además de preferencias, los usuarios elaboran expectativas sobre los resultados que pueden esperar. Y recordando las evidencias presentadas en el apartado del placebo, no es de extrañar que las expectativas previas expliquen hasta el $15 \%$ de la varianza de resultados de los tratamientos (Lambert et al., 1986). Los meta-análisis confirman que, efectivamente, las expectativas medidas en los primeros momentos de una terapia tienen un pequeño pero consistente efecto sobre los resultados (Constantino, Arnkoff, Glass, Ametrano y Smith, 2011).

\section{Actividad durante las sesiones}

Las personas necesitan buscar un sentido a los problemas y considerar nuevas formas de ser y actuar para poner en marcha nuevos intentos de solución. Además, cada uno es el mayor especialista en su propia vida y el más experimentado lidiando con sus particulares dificultades. Pues bien, todo este conocimiento lo traen a consulta los usuarios, y sin duda condiciona la manera de interpretar la información que reciben del terapeuta, y sus decisiones sobre cuál aceptar y cuál rechazar.
La investigación de proceso ha tratado de confirmar este planteamiento utilizando herramientas como el Interpersonal Process Recall (IPR), un sistema de análisis del proceso terapéutico que trabaja con la información que terapeutas y usuarios dan al revisar con un investigador las sesiones grabadas de una terapia. Utilizando este tipo de metodología se concluye que los pacientes: evalúan constantemente el desempeño del terapeuta, tratan de entender su programa de trabajo y de responder a las expectativas que este se crea, y son conscientes de las limitaciones del clínico, así que seleccionan lo que les sirve y desechan el resto (Rennie, 1994).

Un aspecto especialmente preocupante es que los usuarios comunican al terapeuta únicamente una parte de sus reflexiones, tal vez la parte que les parece que encaja mejor con la línea de trabajo que se le propone. Por eso no es de extrañar que cuando se comparan, usando el IPR, las visiones de los clientes y de los terapeutas, sobre un determinado evento de una sesión, las historias no siempre coinciden; ambos protagonistas tienen diferentes interpretaciones para el mismo hecho (Elliott et al., 1994). Cuando clientes y terapeutas revisan sesiones por separado tratando de identificar qué momentos o intervenciones son especialmente significativos para el cambio, el acuerdo entre partes es sólo moderado. En un estudio de Levitt y Piazza-Bonin (2011) en el que cuatro clientes y cuatro terapeutas analizan una sesión de psicoterapia, de los 70 eventos de cambio encontrados, sólo 31 son señalados por terapeuta y cliente; los otros 39 los señala únicamente una de las partes.

Por lo demás parece claro que los usuarios suelen tener sus propias ideas sobre porqué se produce el problema (Wall y Hayes, 2000); y, en general, que el terapeuta las descubra y apoye se relaciona con los buenos resultados de los tratamientos (Hayes y Wall, 1998). Hay también evidencias de que las personas llegan a consulta con sus propias ideas sobre lo que necesitan para mejorar y de que estas ideas van a influir en el tipo de relación que establezcan con sus terapeutas (Philips, Werbart, Wennberg y Schubert, 2007). No es de extrañar, además, que si tienen claro lo que pueda serles de utilidad, sean con frecuencia muy intencionales y dirijan las consultas hacia los temas que estiman más beneficiosos para ellos (Rennie, 2000).

En esta línea es especialmente interesante el estudio llevado a cabo en Dinamarca por Mackrill (2008). Trabajando sobre diarios de sesiones realizados por sus clientes, el autor concluye que: las personas traen a terapia estrategias propias para enfrentarse a sus problemas, las comparan con las propuestas por los terapeutas, y si no coinciden, seleccionan lo que les parece útil de las sugerencias de los profesionales y desechan el resto.

Además, sabemos que los clientes no siempre están de acuerdo con sus terapeutas y que tratan de construir nuevos significados y producir cambios aprovechando incluso los errores de estos (Levitt, Butler y Hill, 2006).

Todo ello apoya la idea de que la psicoterapia debe entenderse como un proceso conjunto de construcción del conocimiento. Los usuarios traen sus ideas y los terapeutas sus 
modelos y su experiencia personal. Obviamente cada persona que busca ayuda es diferente, y cada uno tiene su estilo de colaboración, unos se implican más y otros menos, unos piden terapeutas más directivos y otros prefieren profesionales más flexibles; pero raramente son receptores pasivos. Y este es el último tema a abordar, cómo la implicación en los tratamientos parece ser una variable esencial a la hora de explicar el éxito de una intervención (Orlinsky, Ronnestad y Willutzki, 2004).

Implicación significa disposición para trabajar, apertura para el cambio. No necesariamente encaja con lo que entendemos por motivación, uno puede desear un cambio pero no apetecerle involucrarse activamente en trabajar para conseguirlo. La implicación se desarrolla en el contexto de una buena alianza de trabajo, pero implicación y relación terapéutica son aspectos diferentes. Un cliente se puede sentir muy entendido por su terapeuta pero eso no le lleva necesariamente a convertirse en un agente de cambio activo. Para entender el sentido más interesante de la implicación parece especialmente relevante el concepto de motivación para la autonomia (Zuroff, Koestner, Moskowitz, McBride, Marshall y Bagby, 2007).

La motivación para la autonomía se define como el grado en que los clientes perciben su participación en terapia como libremente elegida. Están ahí porque quieren estar y colaboran de la forma en que quieren colaborar. En un estudio con personas diagnosticadas de depresión, Zuroff et al. (2007) demuestran que la motivación para la autonomía es mejor predictor de resultados que las medidas de alianza terapéutica. Y, lo que es más importante, encuentran que la motivación para la autonomía se desarrolla cuando hay un terapeuta que facilita y promociona que los clientes asuman esa responsabilidad. Este dato parece muy relevante para entender lo que hace exitoso a un terapeuta: no se trata únicamente de conseguir que el usuario se sienta entendido, hay que devolverle además que la responsabilidad para el cambio es suya, y que tiene las competencias necesarias para ponerse en marcha.

\section{E1 trabajo fuera de las sesiones}

Lo que los clientes hacen en el periodo entre sesiones tiene también su impacto sobre los resultados de los tratamientos. De entrada, parece claro que pedir tareas mejora los resultados de los tratamientos (Kazantzis, Whittington y Dattilio, 2010), de manera que el esfuerzo de los usuarios al aplicar nuestras sugerencias en su vida cotidiana parece tener recompensa.

Otros datos apoyan que la elaboración que hacen en sus casas del trabajo en sesión también es relevante para explicar el resultado terapéutico. Por ejemplo, un análisis realizado con personas con un diagnóstico de trastorno de alimentación establece que las personas que entre sesiones piensan más en el terapeuta y en la terapia tienen menos riesgo de fracaso; pero con un matiz, los que recrean los diálogos terapéuticos asociándolos a emociones negativas tienden a te- ner peores resultados (Hartmann, Orlinsky, Weber, Sandholz y Zeeck, 2010). En la misma línea, el estudio de los diarios de pacientes, que se presentaba anteriormente, pone de manifiesto que: los clientes son creativos fuera de las sesiones, se organizan sus propias experiencias terapéuticas, y comparan las ideas que sacan de consulta con las opiniones de personas de su ambiente para acabar extrayendo sus propias conclusiones (Mackrill, 2008).

Resumiendo, se puede concluir que los clientes vienen a terapia con preferencias sobre cómo hay que trabajar, expectativas sobre los resultados que puedan obtener y hasta con iniciativas de cambio ya puestas en práctica. Una vez en la sesión, tienen sus propias explicaciones del problema y estrategias de solución que reajustan a través del contacto con los terapeutas. Son activos entre sesiones, recuerdan éstas, ponen en práctica alguna de las ideas que aprendieron y confirman con otras personas de su entorno las ideas que van sacando. Y, lo más importante, parece establecido que de su grado de implicación depende en gran medida el éxito de los tratamientos.

\section{Conclusiones e implicaciones prácticas}

Los seres humanos estamos diseñados para sobrevivir y adaptarnos, y esta capacidad nuestra es fundamental a la hora de entender el funcionamiento de la psicoterapia. Lo que parece una afirmación obvia y de carácter filosófico, puede además apoyarse con datos empíricos. A lo largo de este trabajo se han revisado investigaciones que demuestran que las personas son capaces de soportar y sobreponerse a situaciones críticas, que se pueden recuperar sin ayuda de trastornos mentales graves, que son capaces de sanar siguiendo programas auto-aplicados con muy poco contacto con un terapeuta, o que son capaces de activar sus recursos biológicos o cognitivos cuando un placebo les hace concebir expectativas de curación. Además, estos seres diseñados para sobrevivir, cuando buscan la ayuda de un psicoterapeuta, pueden actuar de una manera activa y colaborativa. Prueba de ello es que generan expectativas de cambio, tienen preferencias sobre el tipo de ayuda a recibir y, en ocasiones, acuden ya con mejorías a los tratamientos. Los clientes de una terapia evalúan a los terapeutas y el tipo de ayuda que estos ofrecen, se quedan con las ideas que les parecen interesantes y rechazan el resto. Utilizan creativamente la información de las consultas en el periodo entre sesiones, la comparten con sus familias y la usan como palanca para el cambio. Por todo ello, pensar que los usuarios de psicoterapia son seres pasivos, atrapados por sus problemas, que esperan que sus terapeutas les resuelvan sus dificultades es, cuando menos, discutible.

Este cambio en la visión de la figura de los usuarios puede ayudarnos a ajustar el papel de los terapeutas en los tratamientos. Al inicio de este trabajo se recordaba que únicamente el 13\% de la varianza de los resultados terapéuticos se atribuye al peso de los tratamientos. Es posible que algunas personas se sientan un tanto decepcionadas con ese dato, pero no conviene dejarse impresionar por el tamaño de la 
contribución, pues nos haría perder perspectiva sobre su relevancia. La proporción de levadura en la masa de pan es de menos del 3\% y sin embargo resulta crucial para determinar el estado final del producto. De igual manera la intervención de los terapeutas con sus tratamientos debe ser entendida como el catalizador del cambio, no importa tanto el tamaño de su efecto como su importancia en el proceso.

Todo el planteamiento realizado hasta aquí apoya la idea de que las personas son capaces de cambiar y de implicarse activamente en su proceso de terapia. Pero eso no quiere decir que todos los usuarios lo hagan; obviamente hay grandes diferencias entre unos y otros. La cuestión es que si las personas tienen al menos esa potencialidad, lo que a los clínicos e investigadores concierne es establecer qué tipo de prácticas favorecerán su desarrollo. Dicho de otra manera lo que conviene es optimizar la parte que parece depender de los terapeutas para conseguir que los usuarios hagan el resto y los resultados de las intervenciones sean las deseadas. Así que lo primero será establecer con que se corresponde ese $13 \%$ de contribución de la técnica. Un resumen muy conciso sobre los efectos de los tratamientos en los resultados ofrece algunas pistas para ello:

1. Los enfoques terapéuticos no establecen grandes diferencias en cuanto a resultados; esto es, no hay modelos que sean claramente mejores que otros (Wampold, 2001).

2. Sí hay terapeutas que son más eficaces que otros y las diferencias entre profesionales contribuyen más a explicar la varianza de resultados que el modelo de tratamiento que se utilice (Baldwin y Imel, 2013).

3. Un aspecto clave para explicar estas diferencias es la alianza terapéutica (Norcross y Wampold, 2011).

Cada una de estas afirmaciones necesitaría de otro artículo como éste para ser razonada, si se mencionan tan resumidamente aquí es sólo para hacer ver la relevancia que tiene el terapeuta y sus habilidades para establecer una buena alianza de trabajo a la hora de explicar los resultados de las intervenciones. Está muy bien tener evidencias, pero lo que los clínicos necesitan son ideas concretas, ¿qué cambios en la práctica nos invitan a hacer estos datos? A continuación se adelantan esquemáticamente algunas recomendaciones, ava-

\section{Referencias}

Baldwin, S. A., \& Imel, Z. E. (2013). Therapist effects. Findings and methods. In M. Lambert (Ed.), Bergin and Garfield's Handbook of Psychotherapy and Behavior Change (6Th ed., pp. 219-257). New York: Wiley.

Benedetti, F. (2013). Placebo and the new physiology of the doctor-patient relationship. Physiological Reviews, 93(3), 1207-1246. doi: 10.1152/physrev.00043.2012

Benedetti, F., Arduino, C., Costa, S., Vighetti, S., Tarenzi, L., Rainero, I., \& Asteggiano, G. (2006). Loss of expectation-related mechanisms in Alzheimer's disease makes analgesic therapies less effective. Pain, 121(1-2), 133-144. doi: http://dx.doi.org/10.1016/j.pain.2005.12.016

Bohart, A. C., \& Greaves, A. L. (2013). The client in psychoterapy. In M. Lambert (Ed.), Bergin and Garfield's Handbook of Psychotherapy and Behavior ladas por la investigación, que pueden servir para fomentar el protagonismo de los usuarios.

- Si se asume que el cliente debe ser protagonista del proceso conviene elegir técnicas que activen sus recursos y favorezcan su implicación. Los terapeutas deben creer en las posibilidades de sus clientes y a partir de esta posición escuchar y privilegiar sus experiencias e ideas de cambio (Bohart y Tallman, 2010).

- Conviene crear lo más rápidamente posible una fuerte relación terapéutica (Horvarth, Del Re, Flückinger y Symonds, 2011). Y lo que parece contribuir a conseguirlo es que los terapeutas ayuden a sus clientes a simbolizar sus experiencias en palabras, para después devolverles que entienden y validan sus historias (Elliott, Bohart, Watson y Greenberg, 2011). Las cualidades de los terapeutas que favorecen la creación de esta relación de trabajo son la autenticidad y coherencia (Kolden, Klein, Wang y Austin, 2011), y la aceptación incondicional de los usuarios (Farber y Doolin, 2011).

- Otro elemento fundamental es construir metas conjuntamente con los usuarios y controlar su consecución a lo largo del proceso (Tyron y Winograd, 2011).

- Además, es importante ajustarse a los clientes a la hora de decidir cómo trabajar, para ello hay que tener en cuenta sus preferencias sobre el tipo de terapia a recibir, sus teorías explicativas sobre los problemas, sus teorías sobre cómo han de producirse los cambios, su estilo y grado de implicación, y sus valores culturales y religiosos (Bohart y Greaves, 2013).

- La actitud del terapeuta hacia su trabajo es fundamental. Los profesionales comprometidos con la constante mejora de sus prácticas, en disposición de aprender nuevas técnicas y ampliar sus visiones teóricas para ser cada vez más útiles a sus clientes, sufren menos burnout y mantienen su eficacia a lo largo de su carrera (Orlinsky y Ronnestad, 2005).

- Por último, aunque por importancia podría ser lo primero, pedir feedback sobre la marcha del tratamiento ha demostrado ser una práctica clave. Bien directamente o usando escalas, la retroalimentación constante de nuestros clientes nos ayuda a mejorar nuestra práctica y a obtener mejores resultados (Lambert, 2010).

Change. (6th ed., pp. 219-257). New York: Wiley.

Bohart, A. C., \& Tallman, K. (1999). How clients make therapy work: The process of active self-healing. Washington, DC: American Psychological Association.

Bohart, A. C., \& Tallman, K. (2010). Clients: The neglected common factor in psychotherapy. In B. Duncan, S. Miller, B. Wampold y M. Hubble (Eds), The heart and soul of change: Delivering what works in therapy (2nd ed., pp. 83-111). Washington, DC: American Psychological Association.

Calhoun, L. G., \& Tedeschi, R. G. (2006). The foundations of posttraumatic growth: An expanded framework. In L. Calhoun y R. Tedeschi (Eds.), Handbook of posttraumatic growth: Research and practice (pp. 1-23). Mahwah, NJ: Erlbaum. 
Constantino, M. J., Arnkoff, D. B., Glass, C. R., Ametrano, R. M., \& Smith, J. Z. (2011). Expectations. Journal of Clinical Psychology, 67(2), 184-192. doi: http://dx.doi.org/10.1002/jclp.20754

Cuijpers, P., Donker, T., van Straten, A., Li, J., \& Andersson, G. (2010). Is guided self-help as effective as face-to-face psychotherapy for depression and anxiety disorders? A systematic review and meta-analysis of comparative outcome studies. Psychological Medicine, 40(12), 1943-1957. doi: $10.1017 / \mathrm{s} 0033291710000772$

Duncan, B. L. (2010). On becoming a better therapist. Washington, D.C: American Psychological Association.

Duncan, B. L., \& Miller, S. D. (2000). The heroic client: Doing client-directed, outcome-informed therapy. San Francisco, CA: Jossey-Bass.

Elliott, R., Bohart, A. C., Watson, J. C., \& Greenberg, L. S. (2011). Empathy. In J.C. Norcross (Ed.), Psychotherapy relationships that work: Evidence-based responsiveness (2nd ed., pp.132-152). New York, NY: Oxford University Press.

Elliott, R., Shapiro, D. A., Firth Cozens, J., Stiles, W. B., Hardy, G. E., Llewelyn, S. P., \& Margison, F. R. (1994). Comprehensive process analysis of insight events in cognitive-behavioral and psychodynamic-interpersonal psychotherapies. Journal of Counseling Psychology, 41(4), 449-463.

Farber, B. A., \& Doolin, E. M. (2011). Positive regard and affirmation. In J.C. Norcross (Ed.), Psychotherapy relationships that work: Evidence-based responsiveness (2nd ed., pp.168-186). New York, NY: Oxford University Press.

Frank, J. D. (1973). Persuasion and healing: a comparative study of psychotherapy. Baltimore: Johns Hopkins University Press.

Grissom, R. J. (1996). The magical number $.7 \pm .2$ : Meta-meta-analysis of the probability of superior outcome in comparisons involving therapy, placebo, and control. Journal of Consulting and Clinical Psychology, 64, 973982.

Hartmann, A., Orlinsky, D., Weber, S., Sandholz, A., \& Zeeck, A. (2010). Session and intersession experience related to treatment outcome in bulimia nervosa. Psychotherapy, 47(3), 355-370. doi: 10.1037/a0021166

Hayes, J. A., \& Wall, T. N. (1998). What influences clinicians ' responsibility attrubutions? The role of problem type, theoretical orientation, and cliente attribution. Journal of Social and Clinical Psychology, 17(1), 69-74.

Horvarth, A. O., Del Re, A. C, Flückinger, C., \& Symonds, D. (2011). Alliance in individual Psychotherapy. In J.C. Norcross (Ed.), Psychotherapy relationships that work: Evidence-based responsiveness (2nd ed., pp. 25-69). New York, NY: Oxford University Press.

Howard, K. I., Kopta, S. M., Krause, M. S., \& Orlinsky, D. E. (1986). The dose-effect relationship in psychotherapy. American Psychologist, 41, 159164.

Kaptchuk, T. J., Kelley, J. M., Conboy, L. A., Davis, R. B., Kerr, C. E., Jacobson, E. E., . . Lembo, A. J. (2008). Components of placebo effect: randomised controlled trial in patients with irritable bowel syndrome. BMJ, 336(7651), 999-1003.

Kazantzis, N., Whittington, C., \& Dattilio, F. (2010). Meta-analysis of homework effects in cognitive and behavioral therapy: A replication and extension. Clinical Psychology: Science and Practice, 17(2), 144-156.

Kirsch, I. (2005). Placebo psychotherapy: Synonym or oxymoron? Journal of Clinical Psychology, 61(7), 791-791-803.

Kirsch, I. (2010). The Emperor's New Drugs: Exploding the Antidepressant Myth. New York: Basic Books.

Kirsch, I., Deacon, B. J., Huedo-Medina, T. B., Scoboria, A., Moore, T. J., \& Johnson, B. T. (2008). Initial severity and antidepressant benefits: a meta-analysis of data submitted to the Food and Drug Administration. PLoS Medicine / Public Library of Science, 5(2), e45.

Kolden, G. G., Klein, M. H., Wang, Ch., \& Austin, S.B. (2011). Congruence/Genuineness. In J.C. Norcross (Ed.), Psychotherapy relationships that work: Evidence-based responsiveness (2nd ed., pp.187-202). New York, NY: Oxford University Press.

Lambert, M. J. (2010). "Yes, it is time for clinicians to routinely monitor treatment outcome". In B. Duncan, S. Miller, B. Wampold y M. Hubble (Eds.), The heart and soul of change: Delivering what works in therapy (2nd ed., pp. 239-266). Washington, DC: American Psychological Association.

Lambert, M. J., Shapiro, D. A., \& Bergin, A. E. (1986). The effectiveness of psychotherapy. In S. Garfield y A. Bergin (Eds.), Handbook of psychotherapy and behavior change (3rd ed., pp. 157-212). New York: Wiley.

Lawson, D. M. (1994). Identifying pretreatment change. Journal of Counseling
\& Development, 72, 244-248.

Levitt, H. M., Butler, M. \& Hill, T. (2006). What Clients Find Helpful in Psychotherapy: Developing Principles for Facilitating Moment-toMoment Change. Journal of Counseling Psychology, 53(3), 314-314.

Levitt, H. M., \& Piazza-Bonin, E. (2011). Therapists' and clients' significant experiences underlying psychotherapy discourse. Psychotherapy Research, 21(1), 70-85. doi: http://dx.doi.org/10.1080/10503307.2010.518634

Mackrill, T. (2008). Exploring psychotherapy clients' independent strategies for change while in therapy. British Journal of Guidance \& Counselling, 36(4), 441-453. doi: http://dx.doi.org/10.1080/03069880802343837

Masten, A. S., \& Narayan, A. J. (2012). Child Development in the Context of Disaster, War, and Terrorism: Pathways of Risk and Resilience. Annual Review of Psychology, 63, 227.

Menchola, M., Arkowitz, H. S., \& Burke, B. L. (2007). Efficacy of SelfAdministered Treatments for Depression and Anxiety. Professional Psychology: Research and Practice, 38(4), 421-429. doi: 10.1037/07357028.38.4.421

Miller, W. R., \& Carroll, K. M. (2006). Drawing the scene together: Ten principles, ten recommendations. In W. Miller y K. Carroll (Eds.), Rethinking substance abuse: What the science shows, and what we should do about it (pp. 293-312). New York: Guilford Press.

Norcross, J. C. (2006). Integrating self-help into psychotherapy: 16 practical suggestions. Professional Psychology: Research and Practice, 37, 683-693.

Norcross, J. C., \& Wampold, B. E. (2011). Evidence-based therapy relationships: Research conclusions and clinical practices. In J.C. Norcross (Ed.), Psychotherapy relationships that work: Evidence-based responsiveness (2nd ed., pp. 423-430). New York, NY: Oxford University Press.

Orlinsky, D. E., \& Ronnestad, M. H. (2005). How psychotherapist develop. A estudy of therapeutic work and professional growth. Washington, D.C.: American Psychological Association.

Orlinsky, D. E., Ronnestad, M. H., \& Willutzki, U. (2004). Fifty years of psychotherapy process-outcome research: Continuity and change. In M. Lambert (Ed.), Bergin and Garfield's handbook of psychotherapy and behavior change (5th ed., pp. 307-390). New York: Wiley.

Ozer, E. J., Best, S. R., Lipsey, T. L., \& Weiss, D. S. (2003). Predictors of posttraumatic stress disorder and symptoms in adults: A meta- analysis. Psychological Bulletin, 129, 52-71.

Philips, B., Werbart, A., Wennberg, P., \& Schubert, J. (2007). Young adults' ideas of cure prior to psychoanalytic psychotherapy. Journal of Clinical Psychology, 63(3), 213-232. doi: http://dx.doi.org/10.1002/jclp.20342

Rennie, D. L. (1994). Clients' deference in psychotherapy. Journal of Counseling Psychology, 41(4), 427-437. doi: 10.1037//0022-0167.41.4.427

Rennie, D. L. (2000). Aspects of the client's conscious control of the psychotherapeutic process. Journal of Psychotherapy Integration, 10(2), 151-167. doi: http://dx.doi.org/10.1023/A:1009496116174

Skodol, A. E., Bender, D. S., Pagano, M. E., Shea, M. T., Yen, S., Sanislow, C. A., ... Gunderson, J. G. (2007). Positive childhood experiences: Resilience and recovery from personality disorder in early adulthood. Journal of Clinical Psychiatry, 68(7), 1102-1108.

Swift, J. K., Callahan, J. L., \& Vollmer, B. M. (2011). Preferences. Journal of Clinical Psychology, 67(2), 155-165. doi: 10.1002/jclp.20759

Tedeschi, J. T., \& Felson, R. B. (1994). Violence, aggression, and coercive actions. Washington, DC: American Psychological Association.

Tyron, G. S., \& Winograd, G. (2011). Goal consensus and collaboration. In J.C. Norcross (Ed.), Psychotherapy relationships that work: Evidence-based responsiveness (2nd ed., pp.153-167). New York, NY: Oxford University Press.

Vaillant, G. E. (1993). The wisdom of the ego. Cambrigde, MA: Harvard University Press,

Wall, T. N. y Hayes, J. A. (2000). Depressed Clients' attributions of responsibility for the causes of and solutions to their problems. Journal of Counseling \& Development, 78(1), 81-86. doi: 10.1002/j.15566676.2000.tb02563

Wampold, B. E. (2001). The great psychotherapy debate: models, methods, and findings. Mahwah, N.J.: L. Erlbaum Associates.

Werner, E., \& Smith, R. (1989). Vulnerable but invincible. New York: Adams Bannister Cox Pubs.

Whiteford, H. A., Harris, M. G., McKeon, G., Baxter, A., Pennell, C., Barendregt, J. J., \& Wang, J. (2013). Estimating remission from untreated major depression: a systematic review and meta-analysis. Psychological 
Medicine, 43, 1569-1585.

Zolkoski, S. M., \& Bullock, L. M. (2012). Resilience in children and youth: A review. Children and Youth Services Revien, 34(12), 2295-2303.

Zuroff, D. C., Koestner, R., Moskowitz, D. S., McBride, C., Marshall, M., \& Bagby, R. (2007). Autonomous motivation for therapy: A new common factor in brief treatments for depression. Psychotherapy Research, 17(2), 137.

(Articulo recibido: 12-02-2014; revisado: 07-05-2014; aceptado: 09-05-2014) 